\title{
Acrofacial dysostosis, Rodríguez type
}

INSERM

\section{Source}

INSERM. (1999). Orphanet: an online rare disease and orphan drug data base. Acrofacial dysostosis, Rodriguez type. ORPHA:1788

Acrofacial dysostosis Rodriguez type is a multiple malformative syndrome in which mandibulofacial dysostosis and severe limb reduction defects are associated with complex malformations of different organs and systems especially the CNS, urogenital tract, heart, and lungs. The mandibulofacial defect, characterized by extremely severe microretrognathism and cleft palate, causes death by respiratory distress. Limb reduction is severe and includes shoulder and pelvis hypoplasia, phocomelia with humerus hypoplasia, absent radius and ulna, complete absence of long bones of the legs, and various hand anomalies, predominantly preaxial reduction (absent thumbs). Other features include CNS malformations (agenesis of corpus callosum and acqueductal stenosis), lung anomalies (absent lung lobulation), complex cardiac malformations, and unicornis uterus. These infants also show facial dysmorphism and ear anomalies. The condition is a rare with an autosomal recessive mode of inheritance. The prognosis is poor and this condition leads to death in utero or shortly after birth. 